\title{
Fluid Milk Purchase Patterns in the South: Effects of Use of Nutrition Information and Household Characteristics
}

\author{
Kim Jensen*
}

\begin{abstract}
This study measures the impacts of use of nutrition information and household socioeconomic characteristics on market participation and amount purchased of whole-fat and lowfat milks in the South. Data are from the 1987-88 Nationwide Food Consumption Survey. The results showed that use of nutrition information had little effect on purchase levels, but did affect market participation. Results suggest promotion of milk purchases on the basis of nutritional benefits through health professionals and product packaging are useful tools for the dairy industry to attract market participation.
\end{abstract}

Key Words: fluld milk, household purchases, market participation

Household expenditure patterns on wholefat versus low-fat milks have changed markedly over the last several decades. Annual U.S. percapita consumption of whole-fat milk dropped from over 154 pounds in 1978 to less than 103 pounds in 1988. During this same time period, per capita consumption of low-fat milks increased by about 31 pounds (Putnam and Allshouse). Results from past studies have suggested that factors such as an aging of the population, more concern about health and nutrition, increased incomes, and increased education levels have contributed to these changes (Haidacher, Blaylock, and Myers, Heien and Wessells (1988a), Heien and Wessells (1988b), Smith, Herrmann, and Warland, Jensen, Kesevan, and Johnson, Herrmann, Sterngold, and Warland). Results from Herrmann, Sterngold, and Warland's study showed that consumers with high levels of concern about fat and cholesterol intake were more likely to have switched from consuming whole-fat milk than those who did not have high levels of concern. If these concerns are great enough, a household may elect not to participate in the market for whole-fat milk regardless of relative prices or income levels. Hence, in this case, the decision to participate in the market for whole-fat milk may be separate from the decision of how much to spend.

This study measures the impacts of use of nutrition information and household socioeconomic characteristics on market participation and amount purchased of whole-fat and low-fat milks in the South. Sources of nutrition information include information from health professionals, packaging or labels, and media sources. Three models (Cragg Market Participation, Tobit, and Complete Dominance) are examined to determine if use of nutrition information and household characteristic variables affect market participation differently than these variables affect amount purchased of each type of milk. The models are also examined to

\footnotetext{
${ }^{*}$ Kim Jensen is an associate professor in the Department of Agricultural Economics and Rural Sociology at the University of Tennessee, Knoxville.
} 
determine if lack of purchases solely represents nonparticipation in the market.

Studies by Boehm and Huang and Raunikar addressed the relationship between fluid milk consumption patterns in the South and household socioeconomic and demographic characteristics. However, these studies were conducted using data from the 1970s. Spending patterns on whole-fat and low-fat milk have changed since then. In the sample of households from the 1977-78 Nationwide Food Consumption Survey (NFCS) used by Huang and Raunikar, the number of households with expenditures on whole-fat milk was 4.2 times the number of households with expenditures on low-fat milk. In this study (with households from the 198788 NFCS), the number of households with purchases of whole-fat milk is only 1.7 times the number of households with purchases of low-fat milk.

The population of the South also underwent many changes during the decade between 1977-78 and 1987-88.' The population grew from 69.8 million in 1977 to 83 million in 1987 (U.S. Bureau of the Census). The South experienced positive net migration while other regions experienced negative net migration. For example, in 1987 alone, the net migration was 4 million. The characteristics of the population have changed: the proportion of the population comprised by whites is declining, the average size of household declined from nearly 2.9 persons to less than 2.7 persons, and the population is aging. In the sample used by Huang and Raunikar, 76 percent of households which purchased whole-fat milk were white households and 89 percent of those purchasing low-fat milk were white households. In the sample used in this study, about 68 percent of households purchasing whole-fat milk are white, and about 88 percent of households purchasing low-fat milk are white. ${ }^{2}$ In Huang and Raunikar' sample, incomes of households purchasing whole-fat milk were almost 78 percent of those purchasing low-fat milk. In the sample used in this study, incomes of households purchasing whole-fat milk are less than 72 percent of those purchasing low-fat milk.

Boehm's study only included consuming households. The study by Huang and Raunikar used the Tobit specification which does not allow market participation and purchase level decisions to differ. This means that the studies by Boehm and Huang and Raunikar did not consider the possibility that some households may not purchase milk regardless of price and income changes or that factors affecting the market participation decision by households might differ from those affecting the purchase level decision. The effects of nutrition awareness on milk purchase patterns in the region were also not addressed in the studies cited.

Several studies have compared the Tobit model with more flexible specifications including the Cragg Market Participation Model (Haines, Guilkey, and Popkin, Blisard and Blaylock, Blaylock and Blisard, and Gould) and Heckman Models, such as the Complete Dominance Model (Blaylock and Blisard). Market participation models allow the decision of whether to participate in a market to differ from the purchase or consumption level decision. Therefore, zero household purchases on milk could represent either the decision by a household not to participate in the market or a market participant at a corner solution (zero purchases due to income levels or relative prices). The Tobit model assumes that everyone is a market participant and, hence, does not allow for separate market participation and consumption level decisions. Zero purchases are due solely to standard corner solutions. The Complete Dominance Model assumes once a household decides to participate in the market, it will have positive purchase levels. Therefore, zero purchases solely represent lack of participation in the market and do not represent standard corner solutions. The Complete Dominance model is more plausible when factors such health considerations have a marked effect on dividing the market between participants and nonparticipants.

The remainder of this paper is as follows. The Method of Analysis section presents the likelihood functions in logarithmic form from the Cragg Market Participation Model (CMP), Tobit Model, and Complete Dominance Model(CD) and shows how the three models are related. The Data section discusses the NFCS sample and the variables included in the models. In the Results section, likelihood ratio tests used in selecting a model specification for each type of milk are presented. Parameter estimates from the three model specifications are presented for purposes of comparison. Using the estimates from the selected 
models, two profile households are formulated. The effects of nutrition information use on purchase patterns for the two profile households are then projected. The Conclusions section discusses the implications of the results for policymakers, providers of nutritional information, and the dairy industry.

\section{Method of Analysis}

The CMP model is the most general of the three models since it allows zero to represent either lack of market participation or a standard corner solution. The Tobit and CD models are restricted versions of the CMP model. The logarithmic form of the likelihood function for the CMP model is:

$$
\begin{aligned}
\ln L= & \sum_{Y_{i}>0}-1 / 2\left[\ln 2 \Pi+\ln \sigma^{2}\right. \\
& \left.+\left(Y_{l}-\beta^{\prime} X\right)^{2} / \sigma^{2}\right]+\ln \Phi_{l}\left(\alpha^{\prime} Z_{l}\right) \\
& \left.+\sum_{Y_{i}=0} \ln \left[1-\Phi_{2}\left(\beta^{\prime} X_{l}\right) / \sigma\right) \Phi_{l}\left(\alpha^{\prime} Z_{l}\right)\right]
\end{aligned}
$$

where $Y_{1}$ is household i's amount purchased per person of whole-fat or low-fat milk, $\Phi_{1}$ is the cumulative probability of market participation, $\Phi_{2}$ is the cumulative probability of nonzero purchases given market participation, $\sigma$ is the standard error of amount purchased, $X_{t}$ and $Z_{t}$ are socioeconomic variables explaining amount purchased and market participation, respectively (INCOME, URBANIZATION, SOUTH ATLANTIC, EAST S. CENTRAL, CHILDREN 0-14, TEENS 15-20, ADULTS 21-40, ADULTS OVER 40, RACE, EDUCATION, GENDER, NUTRITION INFORMATION-HEALTH PROFESSIONALS, NUTRITION INFORMATION-PACKAGING, NUTRITION INFORMATION-MEDIA). The variables explaining amount purchased $(X)$ may or may not be the same as the variables explaining market participation ( $Z$ ). The $\beta$ and $\alpha$ are parameters associated with purchase level and market participation, respectively.

If all consumers are assumed to be market participants, then the probability of market participation is one, that is, $\Phi_{1}=1$. In this case, the $\log$ likelihood function from equation (1) collapses to the log likelihood function for the Tobit model, so that:

$$
\begin{aligned}
\ln L= & \sum_{Y_{>}>0}-I / 2\left[\ln 2 \Pi+\ln \sigma^{2}\right. \\
& \left.+\left(Y_{i}-\beta^{\prime} X\right)^{2} / \sigma^{2}\right] \\
& +\sum_{Y_{1} \text { ×0 }} \ln \left[1-\Phi_{2}\left(\beta^{\prime} X_{i}\right) / \sigma\right]
\end{aligned}
$$

Equation (2) implies that all zero purchases must represent corner solutions, thus, everyone is assumed to be a market participant.

Alternatively, if the zero purchases solely represent nonparticipation in the market, then the probability of nonzero purchases given market participation is one or $\Phi_{2}=1$. In this case, equation (1) collapses to the log likelihood function for the $C D$ model, which is:

$$
\begin{aligned}
\ln L= & \sum_{Y_{>}>0}-1 / 2\left[\ln 2 \Pi+\ln \sigma^{2}\right. \\
& \left.+\left(Y_{1}-\beta^{\prime} X\right)^{2} / \sigma^{2}\right]+\ln \Phi_{l}\left(\alpha^{\prime} Z_{i}\right) \\
& +\sum_{Y_{i}=0} \ln \left[1-\Phi_{l}\left(\alpha^{\prime} Z_{l}\right)\right]
\end{aligned}
$$

Equation (3) implies that everyone who is a market participant has positive purchase levels of the product. Since the market participation and purchase level decisions differ and all zeroes represent nonparticipation, the log likelihood function of the CD Model is a combination of the probit $\log$ likelihood and the $\log$ likelihood for regression on the nonzero observations. Since (2) and (3) are restricted specifications of (1), likelihood ratio tests for model specification can be conducted.

The probabilities of market participation and nonzero purchases, as well as conditional and unconditional means, can be calculated from the three models. For purposes of brevity, formulas for these measures are presented in table 1. From table 1 , it can be seen that the probability of nonzero purchases is a combination of the probability of market participation and the probability of not being at a standard corner solution (nonzero purchases given market participation). Note that the probability of market participation is assumed to be one in the Tobit model. The probability of nonzero purchases given market participation is assumed to be one in the CD model. The conditional mean is the mean of amount purchased for purchasing 
Table 1. Probabilities of Market Participation and Nonzero Purchases and Conditional and Unconditional Means for the CMP, Tobit, and $C D$ Models

\begin{tabular}{lccc}
\hline Measure & CMP & Tobit & $C D$ \\
\hline $\begin{array}{l}\text { Probability of Market } \\
\text { Participation }\end{array}$ & $\Phi\left(\alpha X_{l}\right)$ & 1 & $\Phi\left(\alpha X_{l}\right)$ \\
$\begin{array}{l}\text { Probability of Nonzero } \\
\text { Purchases Given Market } \\
\text { Participation }\end{array}$ & $\Phi\left(\beta X_{l} / \sigma\right)$ & $\Phi\left(\beta / X_{l} / \sigma\right)$ & 1 \\
$\begin{array}{l}\text { Overall Probability of Nonzero } \\
\text { Purchases }\end{array}$ & $\Phi\left(\alpha^{\prime} X_{l}\right) \Phi\left(\beta X_{l} / \sigma\right)$ & $\Phi\left(\beta X_{l} / \sigma\right)$ & $\Phi\left(\alpha X_{l}\right)$ \\
$\begin{array}{l}\text { Conditional Mean of } \\
\text { Purchases, } E\left(Y / Y_{l}>0\right)\end{array}$ & $\beta X_{l}+\sigma \frac{\phi\left(\beta^{\prime} X_{l} / \sigma\right)}{\Phi\left(\beta^{\prime} X_{l} / \sigma\right)}$ & $\beta X_{l}+\sigma \frac{\phi\left(\beta / X_{l} / \sigma\right)}{\Phi\left(\beta^{\prime} X_{l} / \sigma\right)}$ & $\rho X_{l}$ \\
$\begin{array}{l}\text { Unconditional Mean of } \\
\text { Purchases, } E\left(Y_{l}\right)\end{array}$ & $\Phi\left(\alpha X_{l}\right) \Phi\left(\beta X_{l} / \sigma\right) E\left(Y_{l} / Y_{l}>0\right)$ & $\Phi\left(\beta^{\prime} X_{l} / \sigma\right) E\left(Y_{l} / Y_{l}>0\right)$ & \\
\hline
\end{tabular}

households only. Since the CD model assumes that all zeroes represent nonparticipation, the conditional mean is calculated with $\beta$, from OLS using only purchasing households. The unconditional mean includes all households and is the conditional mean multiplied by the probability of nonzero purchases.

\section{Data}

The data are from the household component of the 1987-88 NFCS conducted by the Human Nutrition Information Service, United States Department of Agriculture. The data were collected from April 1987 through August 1988. Memory recall of the kind, quantity, and cost of foods and beverages used in the household during the seven days prior to the interview were recorded. As part of the NFCS, respondents were also asked to indicate sources from which they had obtained nutrition information during the year prior to their participation in the survey. Potential sources include health professionals, food packages or labeling, and media sources (See table 2). Specific types of information respondents obtained from these sources were not recorded as part of the survey. Total households responding to the survey were 4,495 . This comprised a 38 percent response rate to the survey. Due to the low response, how representative the sample is of the U.S. population has been questioned (Nayga and Capps, GAO). Despite possible sampling problems, the NFCS still represents a comprehensive source of information regarding household characteristics and consumption and patterns. Of the total sample, 1567 households were from the South.

For the purposes of this study, households which did not have at least one member of the household consuming ten or more adjusted meals during the survey week were deleted. Adjusted meals are the number of meals and meal equivalents. Meals eaten away-from-home, skipped meals, and snacks that might substitute for meals are adjusted to a 21 meals per week basis. Households with incomplete records were also deleted from the sample. The total usable sample for the South was 1,430 households. A listing of the specific variables used in the models and their descriptions are presented in table 2 .

As shown in table 3, 34.9 percent of households in the sample have purchases of low-fat milk, while 60.7 percent have purchases of wholefat milk. Only about 4 percent of the sample have purchases of types of milk. The sample means in table 3 reflect differences in the compositions of households with purchases of whole-fat versus lowfat milks. Households with low-fat milk purchases have higher incomes than those without purchases. In contrast, households with whole-fat milk purchases have lower incomes than households without purchases. Differences in urbanization and regional location can be seen between purchasing and non-purchasing households. The age structures of households also differ between purchasing and non-purchasing households. For example, a higher 
Table 2. Variable Names and Definitions

\begin{tabular}{|c|c|}
\hline Variable & Definition \\
\hline \multicolumn{2}{|l|}{ Milk Purchases: } \\
\hline WHOLE-FAT & Household purchases per person of whole-fat milk, pounds \\
\hline LOW-FAT & Household purchases per person of low-fat milk (2 percent milk-fat or less), pounds \\
\hline \multicolumn{2}{|l|}{ Household Characteristics: } \\
\hline INCOME & $\begin{array}{l}\text { Income per person (inciudes before tax earnings from all sources by household } \\
\text { members over age } 15 \text { years for the calendar year prior to the survey interview), } 1000 \\
\text { dollars }\end{array}$ \\
\hline URBANIZATION & 1 if household in a rural area, 0 otherwise \\
\hline \multicolumn{2}{|l|}{ Gcographic Location" } \\
\hline $\begin{array}{l}\text { SOUTH } \\
\text { ATLANTIC }\end{array}$ & $\begin{array}{l}1 \text { if houschold located in Delaware, District of Columbia, Florida, Georgia, } \\
\text { Maryland, N. Carolina, S. Carolina, Virginia, or W. Virginia, } 0 \text { otherwise }\end{array}$ \\
\hline $\begin{array}{l}\text { EAST SOUTH } \\
\text { CENTRAL }\end{array}$ & 1 if household located in Alabama, Kentucky, Tennessee, or Mississippi, 0 otherwise \\
\hline \multicolumn{2}{|l|}{ Age Composition } \\
\hline CHILDREN 0-14 & 1 if children age $0-14$ years present in household, 0 otherwise \\
\hline TEENS 15-20 & 1 if teens and young adults age 15-20 years present in household, 0 otherwise \\
\hline ADULTS $21-40$ & 1 if adults age $21-40$ present in household, 0 otherwise \\
\hline ADULTS OVER 40 & 1 if adults over age 40 present in household, 0 otherwise \\
\hline \multicolumn{2}{|c|}{ Food Manager's Characteristics ${ }^{b}$} \\
\hline$R A C E$ & 1 if food manager is black, 0 otherwise \\
\hline EDUCATION & Education level of food manager, years \\
\hline GENDER & 1 if food manager is female, 0 otherwise \\
\hline \multicolumn{2}{|c|}{ Use of Nutrition Information: } \\
\hline $\begin{array}{l}\text { NUTRITION } \\
\text { INFORMATION- } \\
\text { HEALTH PROF- } \\
\text { ESSIONALS }\end{array}$ & $\begin{array}{l}1 \text { if obtained nutrition information from a doctor, nurse, dietician, home economist, } \\
\text { or extension agent during the year previous to the survey interview, } 0 \text { otherwise }\end{array}$ \\
\hline $\begin{array}{l}\text { NUTRITION } \\
\text { INFORMATION- } \\
\text { PACKAGING }\end{array}$ & $\begin{array}{l}1 \text { if obtained nutrition information from packages or labeling during the year previous } \\
\text { to the survey interview, } 0 \text { otherwise }\end{array}$ \\
\hline $\begin{array}{l}\text { NUTRITION } \\
\text { INFORMATION- } \\
\text { MEDIA }\end{array}$ & $\begin{array}{l}1 \text { if obtained nutrition information from radio, television, newspapers, magazines, or } \\
\text { books during the year previous to the survey interview, } 0 \text { otherwise }\end{array}$ \\
\hline
\end{tabular}

proportion of households which purchase low-fat milks have older adults present (67.5 percent) and do not have children present (70.0) than do households with zero purchases (61.4 percent and 59.8 percent, respectively). The opposite is true for the age structures when compared across whole-fat milk purchase patterns. The race composition and education levels of the household food managers also differ by milk purchase patterns. As shown in table 3, a higher percentage of households with whole-fat purchases have black food managers than do nonpurchasing households. The opposite is true across low-fat purchase patterns. Education levels of food managers are higher among households with low-fat purchases than nonpurchasing households and are lower among households with whole-fat purchases than those without purchases. A slightly lower proportion of households with whole-fat purchases have female food managers than nonpurchasing households.

Of the three types of sources of nutrition information examined, health professionals, packaging, or media sources, the most commonly used type is media sources (including advertising or programs on radio or television or printed materials in magazines, books, or newspapers). However, use of nutrition information differs by purchase patterns on fluid milk. Higher percentages of households with low-fat purchases use nutrition information from one or more of the three sources than do households without low-fat purchases. In contrast, 
Table 3. Sample Means

\begin{tabular}{|c|c|c|c|c|c|}
\hline & \multicolumn{3}{|c|}{ Low-fat Milk: } & \multicolumn{2}{|c|}{ Whole-fat Milk: } \\
\hline & $\begin{array}{c}\text { All } \\
\text { Households }\end{array}$ & Purchasing & $\begin{array}{c}\text { Non- } \\
\text { Purchasing }\end{array}$ & Purchasing & $\begin{array}{c}\text { Non- } \\
\text { Purchasing }\end{array}$ \\
\hline \multicolumn{6}{|l|}{ Household Characteristics } \\
\hline INCOME & 10.189 & 13.541 & 8.393 & 8.426 & 12.912 \\
\hline URBANIZATION & 0.367 & 0.330 & 0.387 & 0.389 & 0.333 \\
\hline \multicolumn{6}{|l|}{ Geographic Location } \\
\hline SOUTH ATLANTIC & 0.562 & 0.539 & 0.575 & 0.569 & 0.551 \\
\hline EAST S. CENTRAL & 0.166 & 0.212 & 0.142 & 0.138 & 0.210 \\
\hline WEST S. CENTRAL & 0.222 & 0.249 & 0.383 & 0.293 & 0.239 \\
\hline \multicolumn{6}{|l|}{ Age Composition } \\
\hline CHILDREN 0.14 & 0.366 & 0.301 & 0.402 & 0.441 & 0.251 \\
\hline TEENS 15-20 & 0.189 & 0.152 & 0.209 & 0.220 & 0.142 \\
\hline ADULTS $21-40$ & 0.522 & 0.449 & 0.562 & 0.579 & 0.434 \\
\hline ADULTS Over 40 & 0636 & 0.675 & 0.614 & 0.601 & 0.689 \\
\hline \multicolumn{6}{|c|}{ Food Manager's Characteristics: } \\
\hline$R A C E$ & 0.237 & 0092 & 0.316 & 0.290 & 0.155 \\
\hline EDUCATION & 11.843 & 12.762 & 11.351 & 11.465 & 12.427 \\
\hline GENDER & 0914 & 0.915 & 0.915 & 0.918 & 0.907 \\
\hline \multicolumn{6}{|l|}{ Use of Nutrition Information } \\
\hline $\begin{array}{l}\text { NUTRITION } \\
\text { INFORMATION- } \\
\text { HEALTH PROF- } \\
\text { ESSIONALS }\end{array}$ & 0.400 & 0.493 & 0.357 & 0.354 & 0.471 \\
\hline $\begin{array}{l}\text { NUTRITION } \\
\text { INFORMATION- } \\
\text { PACKAGING }\end{array}$ & 0.424 & 0.550 & 0.353 & 0.366 & 0.512 \\
\hline $\begin{array}{l}\text { NUTRITION } \\
\text { INFORMATION- } \\
\text { MEDIA }\end{array}$ & 0.486 & 0.608 & 0.424 & 0.422 & 0.585 \\
\hline Number of Observations & 1430 & 499 & 931 & 868 & 562 \\
\hline
\end{tabular}

lower percentages of households with whole-fat purchases use nutrition information from one or more of the three sources than do households without whole-fat purchases.

\section{Results}

The discussion of results is divided into three subsections. First, the results from the models are compared in order to select a model specification for each type of milk. The estimated parameters from the $\mathrm{CD}$, Tobit, and CMP models of whole-fat milk purchases are shown in table 4. Estimates from the models of low-fat milk purchases are displayed in table 5. Second, the coefficients from the model specification selected for each type of milk are discussed in further detail. Third, the estimated coefficients are used in formulating two household profiles. The effects of use of nutrition information on the probability of market participation, probability of nonzero purchases and amount purchased are projected for the two household profiles (table 6).

For both types of milk, the log-likelihood of the CMP Model is smaller in magnitude than the log-likelihood of the Tobit or CD models. The likelihood ratio statistics comparing the CMP model against the Tobit and CD models for whole-fat milk purchases are 110.06 and 214.06 (table 4). The likelihood ratio statistics comparing the CMP model against the Tobit and $\mathrm{CD}$ models for low-fat milk 
Table 4. Estimated Parameters for CMP, Tobit, and CD Models, Whole-Fat Milka,

\begin{tabular}{|c|c|c|c|}
\hline & $\mathrm{CMP}$ & Tobit & $\mathrm{CD}$ \\
\hline $\begin{array}{l}\text { Market Participation } \\
\text { Intercept }\end{array}$ & $\begin{array}{l}0.7371 * \\
(0.4097)\end{array}$ & & ${ }_{(0.2331)}^{0.9413}$ \\
\hline INCOME & $\begin{array}{l}-0.0080 \\
(0.0053)\end{array}$ & & $-0.01133^{* *}$ \\
\hline URBANIZATION & $\begin{array}{c}0.1524 \\
(0.1241)\end{array}$ & & $\begin{array}{c}0.1331 \\
(0.0760)\end{array}$ \\
\hline SOUTH ATLANTIC & $\begin{array}{l}-0.2403 \\
(0.1526)\end{array}$ & & $\begin{array}{l}-0.0878 \\
(0.0850)\end{array}$ \\
\hline EAST S. CENTRAL & $\begin{array}{l}-0.4964 \\
(0.1847)\end{array}$ & & $(0.1112){ }^{-0.417 * *}$ \\
\hline CHILDREN 0-14 & $\underbrace{0.6742}_{(0.2162)}$ & & ${ }_{(0.0905)}^{0.3203}{ }^{* * *}$ \\
\hline TEENS $15-20$ & ${ }_{(0.2092)}^{0.5651}{ }^{* * *}$ & & $\underset{(0.0934)}{0.1666} *$ \\
\hline ADULTS $21-40$ & $\underbrace{0.8031}_{(0.2534)}$ & & $\left.{ }^{0.2589}\right)^{* * *}$ \\
\hline ADULTS OVER 40 & $\begin{array}{c}0.4252 \\
(0.2675)\end{array}$ & & $\begin{array}{c}0.0283 \\
(0.1064)\end{array}$ \\
\hline$R A C E$ & $\begin{array}{l}0.5146 \\
(0.1996)\end{array}$ & & ${ }_{(0.0900)}^{0.2389}{ }^{* * *}$ \\
\hline EDUCATION & $\begin{array}{l}-0.0567 \\
(0.0200)\end{array}$ & & $\begin{array}{l}-0.0439 \\
(0.0130)\end{array} * * *$ \\
\hline GENDER & $\begin{array}{c}0.0994 \\
(0.1569)\end{array}$ & & $\begin{array}{l}-0.0983 \\
(0.1261)\end{array}$ \\
\hline $\begin{array}{l}\text { NUTRITION INFORMATION- } \\
\text { HEALTH PROFESSIONALS }\end{array}$ & $\begin{array}{l}-0.2898 \\
(0.1175)\end{array}$ & & $\left.\frac{-0.2121}{(0.0734)}\right)^{* * *}$ \\
\hline $\begin{array}{l}\text { NUTRITION INFORMATION- } \\
\text { PACKAGING }\end{array}$ & $\begin{array}{l}-0.1110 \\
(0.1322)\end{array}$ & & $\begin{array}{l}-0.1298 \\
(0.0824)\end{array}$ \\
\hline NUTRITION INFORMATION-MEDIA & $\begin{array}{l}-0.1750 \\
(0.1355)\end{array}$ & & ${ }^{-0.1376}{ }^{(0.0831)} *$ \\
\hline Amount Purchased & $\begin{array}{l}8.3863 \\
(0.9195)\end{array}$ & $\begin{array}{l}6.2517 \\
(0.8658)\end{array}$ & $\begin{array}{l}8.1416 \\
(0.6740)\end{array}$ \\
\hline INCOME & $\begin{array}{l}-0.0253 \\
(0.0185)\end{array}$ & 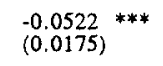 & $\begin{array}{l}-0.0087 \\
(0.0144)\end{array}$ \\
\hline URBANIZATION & $\begin{array}{c}0.4792 \\
(0.3067)\end{array}$ & $\underbrace{0.7019}_{(0.2818)}$ & $\begin{array}{c}0.3641 \\
(0.2179)\end{array}$ \\
\hline SOUTH ATLANTIC & $\begin{array}{c}0.0547 \\
(0.3446)\end{array}$ & $\begin{array}{l}-0.4491 \\
(0.3123)\end{array}$ & $\begin{array}{l}-0.1224 \\
(0.2363)\end{array}$ \\
\hline EAST S. CENTRAL & $\begin{array}{l}-0.6343 \\
(0.4657)\end{array}$ & 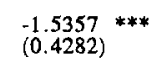 & $\begin{array}{l}-02480 \\
(0.3459)\end{array}$ \\
\hline CHILDREN 0-14 & $\begin{array}{l}-0.7000 \\
(0.3346)\end{array}$ & $\begin{array}{c}0.3595 \\
(0.3299)\end{array}$ & $\begin{array}{l}-0.6619 * * * \\
(0.2450)\end{array}$ \\
\hline TEENS $15-20$ & $\begin{array}{l}-0.4491 \\
(0.3532)\end{array}$ & $\begin{array}{c}0.3455 \\
(0.3360)\end{array}$ & $\begin{array}{l}-0.2279 \\
(0.2486)\end{array}$ \\
\hline ADULTS $21-40$ & $\frac{-1.2102}{(0.3931)}^{* * *}$ & $\begin{array}{c}0.3374 \\
(0.3677)\end{array}$ & $\begin{array}{l}-0.7988 \text { (0** } \\
(0.2746)\end{array}$ \\
\hline ADULTS OVER 40 & $\begin{array}{l}-0.6505 \\
(0.4109)\end{array}$ & $\begin{array}{l}-0.1762 \\
(0.3826)\end{array}$ & $\begin{array}{l}-0.3773 \\
(0.2830)\end{array}$ \\
\hline$R A C E$ & $\begin{array}{l}-1.0435 * * * \\
(0.3791)\end{array}$ & $\begin{array}{c}-.2592 \\
(0.3203)\end{array}$ & $\begin{array}{l}-1.0782 \\
(0.2351)\end{array}$ \\
\hline EDUCATION & $\begin{array}{l}-0.0588 \\
(0.0542)\end{array}$ & $\begin{array}{l}-0.17177^{* * *} \\
(0.0491)\end{array}$ & $\begin{array}{l}-0.0408 \\
(0.0392)\end{array}$ \\
\hline GENDER & $\begin{array}{l}-2.5143 * * * \\
(0.4362)\end{array}$ & $\begin{array}{l}-1.7841 * * * \\
(0.4755)\end{array}$ & $\begin{array}{l}-2.0313 \\
(0.3755)\end{array}$ \\
\hline $\begin{array}{l}\text { NUTRITION INFORMATION- } \\
\text { HEALTH PROFESSIONALS }\end{array}$ & $\begin{array}{l}-0.1445 \\
(0.3049)\end{array}$ & 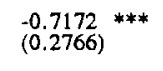 & $\begin{array}{l}-0.0387 \\
(0.2138)\end{array}$ \\
\hline $\begin{array}{l}\text { NUTRITION INFORMATION- } \\
\text { PACKAGING }\end{array}$ & $\begin{array}{l}-0.4097 \\
(0.3309)\end{array}$ & $\begin{array}{l}-0.5999 * \\
(0.3094)\end{array}$ & $\begin{array}{l}-0.2502 \\
(0.2380)\end{array}$ \\
\hline NUTRITION INFORMATION-MEDIA & $\begin{array}{l}-0.0851 \\
(0.3159)\end{array}$ & $\begin{array}{l}-0.4165 \\
(0.3087)\end{array}$ & $\begin{array}{c}0.0080 \\
(0.2340)\end{array}$ \\
\hline Sigma & $\underbrace{3.5944}_{(0.0916)}$ & $\begin{array}{l}4.59555^{* * *} \\
(0.1201)\end{array}$ & \\
\hline Log Likelihood & -2925.07 & -2980.10 & -3032.10 \\
\hline
\end{tabular}


651 Jensen: Fluid Milk Purchase Patterns in the South: Effects of Use of Nutrition Information and Household Characteristics

Table 5. Estimated Parameters for CMP, Tobit, and CD Models, Low-Fat Milke,b

\begin{tabular}{|c|c|c|c|}
\hline Variables & CMP & Tobit & $\mathrm{CD}$ \\
\hline $\begin{array}{l}\text { Market Participation } \\
\text { Intercept }\end{array}$ & 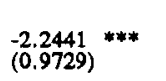 & & $\begin{array}{l}-1.5035 * * * \\
(0.2468)\end{array}$ \\
\hline$I N C O M E$ & ${ }_{(0.0117)}^{0.0453} * * *$ & & $\left.{ }_{(0.0045)}^{0.0135}\right)^{* * *}$ \\
\hline URBANIZATION & $\begin{array}{l}-0.0173 \\
(0.1593)\end{array}$ & & $\begin{array}{l}-0.1157 \\
(0.0786)\end{array}$ \\
\hline SOUTH ATLANTIC & $\begin{array}{c}0.0594 \\
(0.1730)\end{array}$ & & $\begin{array}{l}0.0458 \\
(0.0875)\end{array}$ \\
\hline EAST S. CENTRAL & $\left(_{(0.2251)}^{0.4432}\right.$ & & $\stackrel{0.3893}{(0.1143)}^{* * *}$ \\
\hline CHILDREN O-14 & $\begin{array}{c}0.2069 \\
(0.2089)\end{array}$ & & $\begin{array}{c}0.0297 \\
(0.0935)\end{array}$ \\
\hline TEENS $15-20$ & $\begin{array}{c}0.1524 \\
(0.2315)\end{array}$ & & $\begin{array}{l}-0.1068 \\
(0.0966)\end{array}$ \\
\hline ADULTS $21-40$ & $\begin{array}{l}-0.2213 \\
(0.2302)\end{array}$ & & $\left.\cos ^{-0.2790}\right)^{* * * *}$ \\
\hline ADULTS OVER 40 & $\begin{array}{l}0.1975 \\
(0.2326)\end{array}$ & & $\begin{array}{c}0.0660 \\
(0.1091)\end{array}$ \\
\hline$R A C E$ & $\begin{array}{l}-0.4408 \\
(0.2568)\end{array} *$ & & 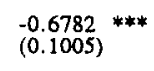 \\
\hline EDUCATION & $\begin{array}{l}0.0785 * * * \\
(0.0248)\end{array}$ & & $\begin{array}{l}0.0636 \\
(0.0138)\end{array}$ \\
\hline GENDER & ${ }_{(0.2145)}^{0.5640}$ & & $\begin{array}{c}0.1705 \\
(0.1321)\end{array}$ \\
\hline $\begin{array}{l}\text { NUTRITION INFORMATION-HEALTH } \\
\text { PROFESSIONALS }\end{array}$ & $\begin{array}{l}0.4148 * * * \\
(0.1550)\end{array}$ & & ${ }_{(0.0765)}^{0.2400}{ }^{* * *}$ \\
\hline $\begin{array}{l}\text { NUTRITION INFORMATION- } \\
\text { PACKAGING }\end{array}$ & $\begin{array}{l}0.37644^{* *} \\
(0.1592)\end{array}$ & & $\underbrace{0.2986}{ }^{(0.0841)}{ }^{* * *}$ \\
\hline NUTRITION INFORMATION - MEDIA & $\begin{array}{l}-0.0580 \\
(0.1652)\end{array}$ & & $\begin{array}{c}0.0179 \\
(0.0858)\end{array}$ \\
\hline $\begin{array}{l}\text { Amount Purchased } \\
\text { Intercept }\end{array}$ & ${ }_{(2.0310)}^{6.3036 * *}$ & ${ }^{-6.4288} * * * *$ & $\left(_{(1.0310)}^{7.0565}\right.$ \\
\hline INCOME & $\begin{array}{l}-0.0192 \\
(0.0191)\end{array}$ & $\left.{ }_{(0.0175)}^{0.0357}\right)^{* *}$ & $\begin{array}{l}-0.0105 \\
(0.0192)\end{array}$ \\
\hline URBANIZATION & $\begin{array}{l}-0.5035 \\
(0.6541)\end{array}$ & $\begin{array}{l}-0.6017 \\
(0.4225)\end{array}$ & $\begin{array}{l}-0.0723 \\
(0.3216)\end{array}$ \\
\hline SOUTH ATLANTIC & $\begin{array}{l}-0.0182 \\
(0.6944)\end{array}$ & $\begin{array}{c}0.3466 \\
(0.4732)\end{array}$ & $\begin{array}{c}0.1669 \\
(0.3681)\end{array}$ \\
\hline EAST S. CENTRAL & $\begin{array}{c}0.6513 \\
(0.9033)\end{array}$ & ${ }_{(0.6039)}^{1.9683} \quad * * *$ & $\begin{array}{c}0.2343 \\
(0.4385)\end{array}$ \\
\hline CHILDREN 0-14 & $\begin{array}{l}-0.5658 \\
(0.8355)\end{array}$ & $\begin{array}{l}-0.2185 \\
(0.4996)\end{array}$ & $\begin{array}{l}-0.4141 \\
(0.3903)\end{array}$ \\
\hline TEENS $15-20$ & $\begin{array}{l}-1.3757 \\
(0.8901)\end{array}$ & $\begin{array}{l}-0.8594 \\
(0.5280)\end{array}$ & $\begin{array}{l}-0.6228 \\
(0.4133)\end{array}$ \\
\hline ADULTS $21-40$ & $\begin{array}{c}1.1754 \\
(0.9276)\end{array}$ & $\begin{array}{l}-1.7400 \\
(0.5694)\end{array}$ & $\begin{array}{l}-0.6236 \\
(0.4486)\end{array}$ \\
\hline ADULTS OVER 40 & $\begin{array}{l}-0.5050 \\
(0.9127)\end{array}$ & $\begin{array}{c}0.1139 \\
(0.5946)\end{array}$ & $\begin{array}{l}-0.3248 \\
(0.4605)\end{array}$ \\
\hline$R A C E$ & $\begin{array}{l}-2.7845 \\
(1.1880)\end{array}$ & $\begin{array}{l}-4.2409 \\
(0.5753)\end{array}$ & $\begin{array}{l}-1.2508 \\
(0.5216)\end{array}$ \\
\hline EDUCATION & $\begin{array}{l}-0.0310 \\
(0.1065)\end{array}$ & $\begin{array}{l}0.3247 \\
(0.0714)\end{array}$ & $\begin{array}{l}-0.0386 \\
(0.0529)\end{array}$ \\
\hline GENDER & $\begin{array}{l}-2.30244^{* *} \\
(0.9729)\end{array}$ & $\begin{array}{c}0.2524 \\
(0.6989)\end{array}$ & ${ }_{(-1.2804}^{(0.5335)}$ \\
\hline $\begin{array}{l}\text { NUTRITION INFORMATION-HEALTH } \\
\text { PROFESSIONALS }\end{array}$ & $\begin{array}{l}-0.0429 \\
(0.5963)\end{array}$ & $\frac{1}{(0.1762}^{* * *}$ & $\begin{array}{l}-0.0035 \\
(0.3024)\end{array}$ \\
\hline $\begin{array}{l}\text { NUTRITION INFORMATION- } \\
\text { PACKAGING }\end{array}$ & $\begin{array}{c}0.0574 \\
(0.5906)\end{array}$ & ${ }_{(0.4543)}^{1.3112}$ & $\begin{array}{l}-0.2941 \\
(0.3421)\end{array}$ \\
\hline NUTRITION INFORMATION-MEDIA & $\begin{array}{c}0.5441 \\
(0.6539)\end{array}$ & $\begin{array}{c}0.2871 \\
(0.4630)\end{array}$ & $\begin{array}{r}0.3783 \\
(0.3551)\end{array}$ \\
\hline Sigma & $\begin{array}{l}4.4738 \\
(0.1924)\end{array}$ & $\underset{(0.2146)}{5.9563}{ }^{* * *}$ & \\
\hline Log Likelihood & -2015.69 & -2041.55 & -2091.99 \\
\hline
\end{tabular}

- * indicates significance at $\alpha=.10, * *$ indicates significance at $\alpha=.05$, and $* * *$ indicates $\alpha=.01$

b Values in parentheses are the standard errors. 
Table 6. Two Household Profiles: Predicted Probabilities and Amount Purchased and the Effects of Use of Nutrition Information

\begin{tabular}{|c|c|c|c|c|}
\hline \multirow[b]{2}{*}{ Measure } & \multicolumn{2}{|c|}{ Profile 1} & \multicolumn{2}{|c|}{ Profile 2} \\
\hline & Whole-fat & Low-fat & Whole-fat & Low-fat \\
\hline \multicolumn{5}{|l|}{$\begin{array}{l}\text { Without Use of Information from Health } \\
\text { Professionals or Packaging: }\end{array}$} \\
\hline Probability of Market Participation & 0.9975 & 0.1163 & 0.2774 & 0.8891 \\
\hline $\begin{array}{l}\text { Probability of Nonzero Purchases Given } \\
\text { Market Participation }\end{array}$ & 0.8273 & 0.6968 & 0.7932 & 0.7990 \\
\hline Probability of Nonzero Purchases & 0.8252 & 0.0810 & 0.2201 & 0.7104 \\
\hline Conditional Purchases (pounds/person) & 4.5018 & 4.5480 & 4.2330 & 5.3217 \\
\hline Unconditional Purchases (pounds/person) & 3.7148 & 0.3686 & 0.9316 & 3.7805 \\
\hline \multicolumn{5}{|l|}{$\begin{array}{l}\text { With Use of Information from Health } \\
\text { Professionals or Packaging: }\end{array}$} \\
\hline Probability of Market Participation & 0.9921 & 0.3437 & 0.1608 & 0.9779 \\
\hline $\begin{array}{l}\text { Probability of Nonzero Purchases Given } \\
\text { Market Participation }\end{array}$ & 0.7850 & 0.6979 & 0.7465 & 0.7999 \\
\hline Probability of Nonzero Purchases & 0.7788 & 0.2400 & 0.1200 & 0.7822 \\
\hline Conditional Purchases (pounds/person) & 4.1746 & 4.5551 & 3.9261 & 5.3302 \\
\hline Unconditional Purchases (pounds/person) & 3.2513 & 1.0926 & 0.4712 & 4.1697 \\
\hline
\end{tabular}

purchases are 51.92 and 152.60 (table 5). The critical value of $\chi^{2}(15 \mathrm{df})_{\alpha=05}$ equals 25.0 . Hence, the null hypotheses that consumers are market participants (Tobit) and that all participants have nonzero purchases (CD) are rejected in favor of the CMP for both whole-fat and low-fat milk purchases.

Several of the estimated parameters in the purchase level portions of the CMP and CD models conflict in sign with those from the Tobit models. These conflicting results may reflect the Tobit model's inability to separate market participation and purchase level effects. The effects of presence of children and young adults in the models of whole-fat milk purchases provide an example. As shown in table 4 , the coefficients carry opposite signs between the purchase level portion of the CMP model and the Tobit model. The coefficients on children and young adults have positive signs in the market participation portion of the CMP and CD models. Also race is insignificant in the Tobit model, but is negative and significant in the purchase level portion of the CMP model. However, race is significant and positive in the market participation portion of the CMP model.
The effects of food managers' gender and education in the models of low-fat milk purchases provide two additional examples (table 5).

Given the results from the likelihood ratio tests, the remaining discussion of the models focuses on the estimated coefficients from the CMP models. Income is not found to significantly affect whole-fat milk purchases. However, income has a significantly positive effect on market participation for low-fat milk. No significant effects of urbanization on whole-fat or low-fat milk purchases are found. The presence of children, teens, or young adults has a positive effect on market participation for whole-fat milk, but a negative effect on purchase levels (children or young adults). Age structure of the household does not significantly affect market participation for low-fat milk. The presence of a black food manager positively affects market participation for whole-fat milk, but negatively affects amount purchased. The presence of a black food manager has negative effects on market participation and amount purchased of low-fat milk. The food manager's education level negatively affects market 
participation for whole-fat milk and positively affects market participation for low-fat milk.

Most of the effects of household and food manager characteristics found in this study are similar to those found in the studies by Huang and Raunikar and Boehm. One conflicting result is the effect of race on whole-fat milk purchases. Results from the previous studies showed that presence of a nonwhite race in the household had a negative effect on both whole-fat and low-fat milk expenditures (or consumption). The results from this study show that the effects differ in sign for market participation versus amount purchased among market participants. Also, unlike Boehm's and Huang and Raunikar's studies, the results from this study indicate whether a characteristic affected market participation, amount purchased, or both. For example, while the effects of education level on expenditures or consumption were examined in the aforementioned studies, market participation effects could not be discerned from expenditure level effects. The results from this study show that education has a significant effect on market participation, but little effect on amount purchased among market participants.

As displayed in tables 4 and 5, the coefficients on the nutrition use variables are all insignificant in the purchase level portions of the models. However, use of nutrition information from health professionals has a significant effect on market participation for whole-fat and low-fat milks. Use of nutrition information from health professionals negatively affects the probability of market participation for whole-fat milk and positively affects probability of market participation for low-fat milk. Use of nutrition information from food packages or labelling also has a positive influence on market participation for low-fat milk. Use of nutrition information from media sources does not significantly influence market participation for either type of milk.

The results in table 4 indicate that households most likely to be market participants for whole-fat milk are those located in the West South Central region, with children, teens, and adults less than forty years old present, with black food managers, with food managers of a low education level, and that do not use nutrition information from health professionals. The results in table 5 suggest that households most likely to be market participants for low-fat milk are those with high incomes, located in the East South Central region, with nonblack food managers, with food managers of a high education level, with female food managers, and that use nutrition information from health professionals and from food packaging.

The effects of using nutrition information from health professionals and packaging are examined for two household profiles. ${ }^{3}$ Given that use of nutrition information primarily affects market participation, not amount purchased, characteristics of the two household profiles are based on their effects on market participation. The profiles are formulated to give contrasting probabilities of market participation. Therefore, the effects of use of nutrition information in households with a low probability of market participation (due to household characteristics) can be compared with the effects in households with a high probability of market participation.

The characteristics of the Profile 1 household are selected such that this household is more likely to be a market participant for whole-fat milk than the Profile 2 household. The characteristics of the Profile 2 household are selected so that this household is more likely to be a market participant for low-fat milk than the Profile 1 household. The Profile 1 household is assumed to have an income of $\$ 6,000$ per person per year, to be located in a nonrural area of the West South Central region, with children, teens, young adults, and older adults present. The Profile 1 household has a black person managing the household food supplies. The household food manager has a high school education ( 12 years) and is male. The Profile 2 household has an income of $\$ 20,000$ per person per year, is located in a nonrural area of the East South Central region, has no children, teens, or young adults present, but does include adults over 40 . The person managing the food supply is a race other than black, has some postgraduate college education (18 years of education total), and is female. In the two base profiles, only nutrition information from media sources is assumed to be used. Predicted values for the two base households are presented in the upper portion of table 6 . 
The predictions in table 6 show the effects of differing household profiles on market participation and amount purchased. For whole-fat milk, the Profile 1 household has a predicted probability of market participation of 0.9975 and a predicted overall probability of nonzero purchases of 0.8252 . In contrast, the Profile 2 household only has a projected 28 percent chance of participating in the market for whole-fat milk and a 22 percent chance of nonzero purchases. For low-fat milk, the Profile 1 household has less than a 12 percent chance of market participation and only an 8 percent chance of nonzero purchases. However, the Profile 2 household has a 71 percent chance of nonzero purchases of low-fat milk. The Profile 1 household is projected to consume 2.7832 pounds more whole-fat milk per person than the Profile 2 household. For low-fat milk, there is a 3.4119 pounds per person difference in purchases between the Profile 2 household and the Profile 1 household.

In the lower portion of table 6 , predictions for the two households are shown given use of nutrition information from health professionals and from packaging. Use of nutrition information from health professionals and packaging sources increases purchases of low-fat milk and decreases purchases of whole-fat milk. However, the magnitude of the changes are quite different between the Profile 1 and Profile 2 households. The probability of market participation for low-fat milk by the Profile 1 household increases from 0.1163 to 0.3437 , while the probability of participation by the Profile 2 household increases from 0.8891 to 0.9779 . For the Profile 1 household, unconditional purchases of lowfat milk increase from .3686 pounds to 1.0926 , almost a 200 percent increase. Unconditional purchases by the Profile 2 household increase by around 10 percent. Use of nutrition information decreases the probability of market participation for whole-fat milk only slightly in the Profile 1 household. However, for the Profile 2 household, the probability drops from 0.2774 to 0.1608 . For the Profile 1 household, unconditional purchases of whole-fat milk drop from 3.7148 pounds to 3.2513 pounds per person, a decrease of about 12 percent. For the Profile 2 household, the decrease in purchases is around 49 percent.

Note that if the Tobit model had been used in generating the predicted probabilities, a market participation probability of one would have been assumed. This assumption is quite different from the Profile 1 household's probability of market participation for low-fat milk. If the CD model had been used to predict the probabilities, the probability of nonzero purchases given market participation would have been assumed to be one. All of the projected values for whole or low-fat milks are below .83 .

\section{Conclusions}

Rejection of the Tobit versus the CMP models for whole-fat milk and low-fat milk purchases suggests that the decision of whether to participate in the market is separate from the purchase level decision by participating households. Zero purchases of whole-fat milks may reflect attitudes about health concerns regarding intake of fat and cholesterol. The signs and significance of parameters on education and use of nutrition information in the CMP model tend to support this hypothesis. However, because the CD model was rejected, zero purchases do not solely represent nonparticipation in the market.

While past studies have examined consumption or expenditures on milk in the region, these studies were did not address the market participation decision separately from the decision regarding the amount purchased. This limited the ability of these studies to identify how household characteristics affect consumption or expenditures. Many of the household characteristics had significant effects in the market participation portion of the models, but not the purchase level portion (and vice versa), and in some cases the effects of household characteristics were opposite in sign across the two portions of the CMP models. If the market participation decisions and purchase level decisions had not been allowed to differ, as with the Tobit model, this would have produced biased estimates. Furthermore, the effects of use of nutrition information on milk purchases were not examined in past studies. With increased interest in health and nutrition among consumers, identifying how nutrition information affects milk purchases is of importance.

The results suggest that nutrition information contained in television or radio programming or in magazines, newspapers, or books has little influence on amount purchased for whole- 
fat or low-fat milks. A possible interpretation of this result is that media sources of nutrition information are not very effective at altering milk purchase patterns. Use of nutrition information from health professionals, such as physicians, dieticians, or extension personnel have significant effects on market participation for both types of milk. This type of nutrition information lowers market participation for whole-fat milk, but increases market participation for low-fat milk. The results also suggest that packaging or labeling may provide an effective source of information for influencing consumers to purchase low-fat milk. Although use of nutrition information did effect market participation, it had no significant effect on amount purchased. Therefore, nutrition information may be useful in altering market participation, but it has little influence on the amount that participating consumers purchase.

An important caveat should be made in interpreting the nutrition information results. Use of nutrition information from sources where the costs of the information search may be relatively high (i.e. visiting a physician or taking time to read many food labels during a shopping trip) have significant effects. Nutrition information from media sources, which are often more readily obtained (i.e. watching a television advertisement) do not have significant effects. This implies that the findings could reflect consumers' attitudes toward nutrition rather than effectiveness of the various types of nutrition information. For example, it is likely that individuals using nutrition information from health professionals have made a concerted effort to obtain the information or have received advice due to a particular health concern. Therefore, these individuals may view themselves or other household members as being at a high risk for health problems due to poor nutrition habits. Similarly, those food managers who take time to read food packages or labels for nutrition information may be those who are most concerned about lowering fat intake while maintaining adequate calcium intake.

Large differences in projected effects of use nutrition information across the two household profiles suggest targeting of nutrition information programs. The projections show that nutrition information programs would have a greater impact on increasing market participation for low-fat milk in households with the characteristics of Profile 1 than on those of Profile 2. However, nutrition information programs would have a greater impact on decreasing market participation for whole-fat milk in households with the characteristics of Profile 2 than on those of Profile 1. One possible explanation for this result may be the strong positive effects of the presence of children and teens on market participation for whole-fat milk. Consumption of whole-fat milk is still often recommended for young children. Notably, the Profile 2 household did not have children or teens present, but did have older adults present.

The results suggest promotion of milk purchases on the basis of nutritional benefits through health professionals and product packaging are useful tools for the dairy industry to attract market participation. Among households that are not likely to be market participants for low-fat milk, use of nutritional information does little to decrease purchases of whole-fat milk, but results in large increases in purchases of low-fat milk. Hence, promotional campaigns might be targeted toward demographic groups that are not already likely to be market participants for low-fat milk. These households include those located in the West South Central region, with low incomes, with food managers with low education levels, with black food managers, or with male food managers.

\section{References}

Blaylock, J. and N. Blisard. "U.S. Cigarette Consumption: The Case of Low-Income Women," Amer, J. Agr. Econ. 74(1993): 698-705.

Blisard, N. and J. Blaylock. "Distinguishing Between Market Participation and Infrequency-of- PurchaseModels of Butter Demand," Amer. J. Agr. Econ. 74(1993): 314-320. 
Boehm, W.T. "The Household Demand for Major Dairy Products in the Southern Region," South. J. Agr. Econ. 7(1975): 187-196.

Cragg, J. "Some Statistical Models for Limited Dependent Variables with Applications to Demand for Durable Goods," Econometrica. 39(1971): 829-844.

Gould, B.L. "At-home Consumption of Cheese: A Purchase Infrequency Model," Amer. J. Agr. Econ. 74(1992): 453-459.

Gould, B.L., T.L. Cox, and F. Perali, "The Demand for Fluid Milk Products in the U.S.: A Demand Systems Approach," West. J. Agr. Econ., 15(1990):1-12.

Haidacher, R.C., J. R. Blaylock, and L. H. Myers, Consumer Demand for Dairy Products, Agr. Econ. Rpt. No. 586, U.S. Dept. of Agr., Econ. Research Service, Washington D.C., March 1988.

Haines, P., D. Guilkey, and B. Popkin. "Modeling Food Consumption Decisions as a Two-Step Process," Amer. J. Agr. Econ. 70(1988): 543-552.

Heckman, J. "Sample Selection Bias as a Specification Error," Econometrica. 47(1979): 153-161.

Heien, D.M. and C.R. Wessells, "The Demand for Dairy Products: Structure, Prediction, and Decomposition," Amer. J. Agr. Econ. 70(1988): 219-228.

Heien, D.M. and C.R. Wessells, "The Nutritional Impact of the Dairy Support Price Program," The Journal of Consumer Affairs. 22(1988): 201-219.

Herrmann, R.O., A. H. Sterngold, and R.H. Warland, Consumers' Shift Toward Lower Fat Dairy Products: 1990, Pennsylvania State University Department of Agricultural Economics and Rural Sociology, Res. Rpt. No.10, May 1991.

Huang, C.L. and R. Raunikar, "Household Fluid Milk Expenditure Patterns in the South and United States," S. J. Agr. Econ. 15(1983): 27-33.

Jensen, H.H., T. Kesevan, and S.R. Johnson, Measuring the Impact of Health Awareness on Food Demand," Rev. Agr. Econ. 14(1992):299-312.

Nayga, R. and O. Capps, "Determinants of Food Away from Home Consumption: An Update," Agribusiness. 8(1992): 549-559.

Putnam, J.J. and J.E. Allshouse, Food Consumption, Prices, Expenditures, 1970-1992, Statistical Bulletin No. 867, U.S. Department of Agriculture, Economic Research Service, Washington D.C., September 1993.

Smith, B.J., R.O. Herrmann, and R.H. Warland, Milk Consumption and Consumer Concerns About Fat, Cholesterol, and Calories, Pennsylvania State University Department of Agricultural Economics and Rural Sociology, Res. Rpt. No. 8, February 1990.

Tobin, J. "Estimation of Relationships for Limited Dependent Variables," Econometrica. 26(1958): 24-36.

U.S. Bureau of the Census, Statistical Abstract of the United States: 1990 (110th edition.), Washington, DC, 1990. 
U.S. General Accounting Office, Nutrition Monitoring: Mismanagement of Nutrition Survey Has Resulted in Questionable Data, GAO, Washington, D.C., July, 1991.

\section{Endnotes}

1. The South includes the South Alantic states (Delaware, District of Columbia, Florida, Georgia, Maryland, N. Carolina, S. Carolina, Virginia, W. Virginia), the East South Central states (Alabama, Kentucky, Tennessee, Mississippi), and the West South Central states (Arkansas, Louisiana, Oklahoma, and Texas).

2. These changes reflect a shift in the racial mix of the population and changes in consumption levels. During the 1970's and 1980's, per capita consumption of other beverages, such as soft drinks and fruit juices increased while overall (whole and low-fat) milk per capita consumption fell (See Putnam and Allshouse).

3. Due to the presence of discrete explanatory variables, calculating predicted probabilities and expenditures at the sample means of the explanatory variables is inappropriate. For example, according to the variable specification for URBANIZATION, a household must either be located in a rural area or a nonrural area, not .367 in a rural area. An alternative to using sample means is to make projections for specific household profiles. 